\title{
EVOLUCIÓN DEL PROGRAMA MÚSICA LOS VIERNES EN LAS AULAS HOSPITALARIAS DE LA REGIÓN DE MURCIA
}

\section{EVOLUTION OF THE PROGRAM MÚSICA LOS VIERNES IN THE HOSPITAL CLASSROOMS OF THE REGION OF MURCIA}

\author{
Aldara Gambao González \\ aldara.gambaog@um.es \\ Alba María López Melgarejo \\ albamaria.lopez@um.es \\ ISEN Centro Universitario. Universidad de Murcia (España)
}

Recibido: $15 / 10 / 2020$

Aceptado: 30/06/2021

\begin{abstract}
Resumen:
Este trabajo tiene como objetivo realizar una revisión histórica que permite dar a conocer la evolución del programa Música los Viernes desarrollado en dos hospitales de la Región de Murcia. Se trata de una investigación cualitativa y recurre tanto al análisis documental como a las entrevistas abiertas. Los resultados obtenidos evidencian los beneficios que aportan el proyecto musical a nivel emocional en el alumnado hospitalizado y muestran como el proceso de institucionalización del mismo avalado durante sus dieciséis años de vigencia. Se ha determinado la tipología de actividades realizadas, los recursos materiales y el lugar de realización. A su vez, se ha observado un incremento significativo en el número de participantes de manera progresiva superando los ochenta maestros durante el último curso. Como conclusión, el programa de música ayuda a solventar los problemas emocionales y a reducir el estrés de los alumnos debido a la hospitalización.
\end{abstract}

Palabras clave: Educación Musical; Educación alternativa; Proyecto educativo; Hospital.

\begin{abstract}
:
This work aims to carry out a historical review that allows to publicize the evolution of the program Música los Viernes developed in two hospitals in Murcia. It is a qualitative research using both documentary analysis and open interviews. The results show the benefits of the project and the music at the emotional level in the hospitalized students as well as the institutionalization process of the same endorsed with sixteen years since its origin. The typology of activities carried out, the material resources and the place of implementation have been determined. There has been a significant increase in the number of participants surpassing eighty teachers during the last year. In conclusion, the music program helps to solve emotional problems and reduce stress for students due to hospitalization.
\end{abstract}

Keywords: Music education; Alternative education; Educational projects: Hospitals. 


\section{Introducción}

La iniciativa Música los Viernes se enmarca dentro del proyecto "Emocionarte" que pretende introducir en las aulas hospitalarias de la Región de Murcia los lenguajes artísticos (plástico y musical). Este proyecto persigue paliar algunos de los problemas emocionales que surgen en los niños hospitalizados mediante el lenguaje musical y plástico. Se busca adaptar el aprendizaje del alumno y, en consecuencia, los contenidos del currículo, teniendo en cuenta el estado de salud del niño, para que dicho proceso de enseñanza sea significativo y puedan utilizarlo en otros contextos de forma independiente. "Emocionarte" surge en el año 2003 y su principal objetivo se orienta a mitigar la angustia y el estrés en los niños hospitalizados. El programa de Música los Viernes aporta a los participantes beneficios no solo educativos, sino también emocionales y sociales, a través del conocimiento de nuevos conceptos de educación musical, la relajación, la socialización con otros compañeros que se encuentran en una situación similar, entre otros.

Este estudio analiza la evolución que ha tenido el programa de Música los Viernes desde su inicio en 2004 hasta el año 2020 que se imparte en las aulas hospitalarias de la Región de Murcia. Para ello, se ha recurrido al paradigma de la investigación cualitativa y al empleo del análisis documental. Se han tenido en cuenta diferentes fuentes de información (entrevistas y análisis de documentos) que permiten conocer desde los materiales empleados a los instrumentos, tipo de actividades realizadas, caracterización de los voluntarios participantes en el proyecto, organización y gestión.

La realización de este estudio surge de la necesidad de reflexionar sobre la evolución que ha tenido el programa de música desde su comienzo hasta hoy en día, cómo surgió la idea del proyecto, conocer los objetivos que persigue, los beneficios que aporta tanto a docentes como al alumnado, su organización (las reuniones, repartición y temática de las sesiones, permisos necesarios, entre otros), conocer el tipo de recursos e instrumentos empleados, número de maestros participantes, si las sesiones se realizan en grupo o de forma individual, las instalaciones de las que dispone el hospital para el aula, las características del alumnado, el tipo de actividades musicales que se realizan, lugar de la realización de las sesiones, cómo se evalúa el proyecto y las sesiones, así como, las problemáticas surgidas. De esta manera, se pretende clarificar, mostrar y dar a conocer una buena práctica que engloba la música dentro del ambiente hospitalario infantil avalada por más de dieciséis cursos escolares.

\subsection{Las aulas hospitalarias: definición y características}

Las Aulas Hospitalarias se sitúan en los centros hospitalarios con el fin de que cualquier niño ingresado por el motivo que fuere tenga la posibilidad de continuar su formación escolar. Tal y como señala García (2012), el Aula Hospitalaria surge para que los niños hospitalizados prosigan con su educación y hallen en ella un lugar recreativo donde poder asistir cuando no se encuentren recibiendo asistencia médica. A su vez, pretenden mejorar la calidad de vida de los mismos durante el periodo que dure la hospitalización. Como afirma Vila y Barrena (2012) dan respuesta a las diversas necesidades que provoca la enfermedad, además de influir tanto en el ámbito familiar, escolar y social del alumnado. También favorece el proceso de socialización evitando la marginación durante el proceso de hospitalización y contribuye a que se desarrolle el proceso de enseñanza-aprendizaje de manera eficaz facilitando su reincorporación a la institución educativa de un modo satisfactorio una vez abandonado el hospital (Caballero, 2007). 
Forman parte del personal que desempeña su labor en este tipo de aulas el maestro y el personal sanitario como médicos y enfermeros así como psicólogos y otras personas voluntarias (Serradas, 2010). Si bien, se debe involucrar en las tareas a las familias del alumnado dado aportan tranquilidad y les acercan a su vida normal (Cárdenas y López, 2005).

El personal docente se encarga de diseñar y organizar los materiales y las actividades que realizan los alumnos. Estas deben ser diseñadas para que se puedan adaptar al día a día de las clases ya que por el estado de ánimo de los niños y/o tratamientos, surgen dificultades para poder hacerlas todas tal y como se plantean desde un principio. Es aconsejable que se promuevan situaciones que favorezcan la comunicación para que los alumnos/as puedan expresarse a través de las diferentes actividades y juegos. Además de acordar cómo se va a trabajar en el aula, hay que tener en cuenta que todo aquello que se realice en la misma pueda ser practicado por los niños desde una cama (Caballero, 2007). Con respecto al papel que tiene el maestro en este contexto, consiste en proporcionar una educación y orientación al alumno hospitalizado y su familia. A su vez, debe mantener contacto con el personal médico y sanitario del hospital de manera continuada, así como con el profesorado del centro educativo de referencia en el que está escolarizado el niño para conocer el punto de partida e informar de la evolución del proceso de enseñanza-aprendizaje (Fernández, 2000).

Entre los aspectos metodológicos a tener en cuenta a la hora de desarrollar la labor docente en un aula hospitalaria el juego debe ser primordial al favorecer la reducción de los niveles de estrés, angustia, tristeza e intranquilidad (Ariza, 2014). De esta forma, los niños son capaces de desconectar y olvidar temporalmente los problemas que les inquietan mientras pasan momentos agradables con sus compañeros y maestros (Mendoza, 2019). Todas estas experiencias ayudan al alumnado a paliar sus miedos relacionados con esta situación extraordinaria, la medicación, los tratamientos e incluso, la muerte (Carrasco, 2008).

Haciendo una revisión legislativa, fue en 1982 cuando se publica por primera vez en España una norma que regula las Aulas Hospitalarias: Ley 13/1982, de 7 de abril, de integración social de los minusválidos. Tres años después se daría luz verde al Real Decreto 334/1985, de 6 de marzo. En la década de los noventa, el Real Decreto 696/1995, de 28 de abril, de ordenación de la educación de los alumnos con necesidades educativas especiales, en su artículo 3.6 estableció que el Ministerio de Educación y Ciencia sería el encargado de fomentar la creación de las aulas hospitalarias tanto en los hospitales como en los centros de rehabilitación como ya se había establecido anteriormente en la normativa previa. Por último, el Real Decreto 1/2013, de 29 de noviembre, por el que se aprueba el Texto Refundido de la Ley General de derechos de las personas con discapacidad y de su inclusión social recoge prácticamente lo mismo que se publicó casi treinta años antes en el Real Decreto 334/1985. A modo de resumen, se puede concluir que la regulación de las aulas hospitalarias en España apenas ha variado desde que se reguló por primera vez en la década de los ochenta.

Junto a la asistencia recibida en las aulas, con frecuencia se promueve la realización de programas educativos, didácticos o lúdicos de los que se benefician los menores hospitalizados al margen de la propia actividad formativa diaria. Dichos programas no persiguen el avance de los contenidos curriculares correspondientes sino que pretenden amenizar la estancia del alumnado y aliviar el estrés que produce el periodo de hospitalización. Los objetivos y temáticas de dichos proyectos son muy variados: TIC (Serrano y Prendes, 2015), Arte (Riquelme, Cerdán y Sánchez, 2012), Música (Barros et al., 2019; Drake, Ben-Yelun y Del Olmo, 2017) e incluso terapia con animales (Jacobsen, 1997; Peña, 2015).

Como se ha mencionado, la Región de Murcia desarrolla dentro de dos de sus hospitales (Hospital Virgen de la Arrixaca y Hospital Santa Lucia) un proyecto denominado Música los Viernes. Este estudio tiene como objetivo ser una revisión histórica de dicho proyecto desarrollado en el hospital Virgen de la Arrixaca y en el Hospital General Universitario Santa 
Lucía de la mencionada comunidad autónoma. Al mismo tiempo, se analiza la organización del proyecto (objetivos, espacios, tipo de actividades musicales, instrumentos empleados, profesorado participante) y se dan a conocer los aspectos burocráticos que vertebran el proyecto.

\section{Método}

Para dar respuesta al objetivo de este estudio se optó por un diseño de investigación cualitativa. Se entiende por investigación cualitativa (1994) cuando los investigadores analizan e interpretan la información que se da en contextos producidos en situaciones reales (Denzin y Lincoln, 1994). Se han establecido cuatro fases para el desarrollo de este trabajo: 1) la reflexiva, donde se acota el problema, 2) el trabajo de campo, momento en el que el investigador recoge la información necesaria para su estudio, bien sea con participantes o en diferentes contextos, 3) analítica, se analizan los datos recogidos previamente y se obtienen unos resultados y conclusiones, 4) informativa, momento en el que se transmiten y se comunican los datos obtenidos (Rodríguez, Gil y García, 1999).

Se recurrió tanto al análisis documental como a la entrevista. En relación con el primero, el análisis documental supone la acción de mostrar la información de distintos documentos con el fin de favorecer su búsqueda o crear un archivo similar que sirva de suplente (Clauso, 1993). Se escogió esta técnica dado que se han consultado un total de 1123 archivos digitales que facilitó previamente el coordinador del proyecto entre las que figuran: las memorias del programa, circulares, fotos, partituras, vídeos, archivos de audio y evaluaciones del profesorado participante, con fecha de creación desde 2004 a 2020. A su vez, se analizó toda la información publicada en Blog del Equipo de Atención Educativa Hospitalaria y Domiciliaria Región de Murcia (Webmaster Plataforma, 2011). Para ello, se recurrió a la creación de dos fichas de recogidas de información creadas ad hoc para esta investigación.

Por otro lado, se entiende por fuentes orales las personas que participan y aportan información fiable y directa para una investigación (Arias, 2019). Por ello, se realizaron dos entrevistas a dos perfiles muy diferenciados con el fin de verificar la información extraída: Roberto Pujol (R), coordinador del programa en ambos hospitales en los que se desarrolla el proyecto y Natalia Monreal $(\mathrm{N})$, maestra-participante con una mayor colaboración en el hospital Virgen de la Arrixaca de Murcia por serle más cercano a su residencia habitual. De acuerdo a cada rol, se elaboraron una serie de preguntas diferenciadas sobre las siguientes dimensiones: 1) Evolución del proyecto, 2) Objetivos, 3) Beneficios, 4) Organización y voluntarios participantes, 5) Recursos, 6) Instalaciones, 7) Alumnado, 8) Sesiones, 9) Evaluación y 10) Problemáticas.

\section{Análisis y discusión de resultados}

\subsection{Los primeros pasos del proyecto}

El programa Música los Viernes se inicia en el año 2004. La idea surge cuando el actual coordinador, Roberto Pujol Sáez, asiste a un curso ofertado por la Consejería de Educación de la Región de Murcia para conmemorar la muerte del escritor de cuentos Has Christian Andersen. En ese momento conoce a los actuales maestros del aula hospitalaria, entre ellos, la actual directora Ana María Ferrer Mendoza. Allí le sugieren ir al aula hospitalaria para dar clases. Para ello, el actual coordinador tuvo que pedir permiso en el centro educativo donde trabajaba (R: de una manera extraoficial de faltar algunos viernes cada trimestre). Ese curso escolar acude tres veces por trimestre al hospital para dar clases de música y al ver que la experiencia es enriquecedora y le gusta la experiencia surge el proyecto en sí (R: Pero en 
realidad al principio surgió un poco sin saber a lo que íbamos, un poco a la aventura). Así pues, se presenta como proyecto a la Consejería de Educación de la Región de Murcia y es aprobado participando en un segundo curso quince personas solo en el Hospital Virgen de la Arrixaca (actualmente se desarrolla también en el Hospital Santa Lucía de Cartagena los jueves).

Por un lado, en relación con cuándo fue la primera vez que Roberto dio clase por primera vez en el hospital fue en el año 2004. En ese año el aula hospitalaria no cuenta aún con ningún tipo de recurso material vinculado a la práctica musical como pueden ser instrumentos escolares ( $R$ : entonces no había ni instrumentos, ni había nada en el hospital, ahora ya se ha ido creando material y se ha ido comprando cosas).

Atendiendo a las memorias del aula, los objetivos que persigue el programa son los siguientes:

- Mitigar la tristeza y ansiedad que se produce en el niño hospitalizado.

- Reconocer las cualidades del sonido en los distintos ejercicios que se realicen en el aula.

- Sentir curiosidad por todos los sonidos. Diferenciar entre ruido y silencio.

- Reconocer los aspectos básicos del ritmo: pulso y acento.

- Aprender canciones por imitación, así como algunos juegos musicales infantiles.

- Interiorizar y ejecutar ritmos sencillos.

- Desarrollar la capacidad de exploración de las posibilidades expresivas del cuerpo y el movimiento.

- Discriminar el timbre de los instrumentos de Percusión Altura Indeterminada y utilizarlos en algunas de las actividades realizadas en clase.

- Practicar la audición activa de obras musicales.

- Dar la posibilidad a los niños hospitalizados de escuchar y ver actividades musicales preparadas por otros niños, posibilitando así su desconexión por un tiempo del entorno hospitalario.

Por otro lado, según la respuesta que dio la maestra entrevistada, Natalia Monreal, el objetivo principal es llevar la música a aquellos niños que están hospitalizados y sobre todo ver gente nueva, caras nuevas, con actividades nuevas e intentar evadirle en la medida de lo posible, tanto a los padres como a ellos, con cosas divertidas, con instrumentos, con cosas que normalmente ellos no hacen en su día a día. Además, el coordinador destaca que el principal objetivo es mitigar la ansiedad de los niños, coincidiendo así con el primer objetivo que se menciona en las memorias y con el que coinciden numerosos autores (Carrasco, 2012; Todres, 2006; Barrera, Rykov y Doyle, 2002) (R: el proyecto tiene muchos objetivos, algunos muy ambiciosos, pero el primer objetivo del proyecto, que a mí me gusta decirlo bien claro y fuerte, es mitigar la ansiedad que se produce cuando un niño está hospitalizado).

Al ser el principal objetivo del proyecto mitigar la tristeza y ansiedad que se produce en el niño hospitalizado, se antepone el aspecto emocional frente al académico. Asimismo, al ser un docente diferente el que acude cada viernes, el alumnado conoce a distintos profesionales que le muestran actividades variadas ( $\mathrm{N}$ : sobre todo distintas actividades y distintas cosas de forma de ver la música).

Por otro lado, los beneficios que aporta el programa a los maestros que participan en él es la satisfacción de poder ayudar a los niños que se benefician del proyecto (R: a veces uno piensa que donde realmente hace falta es donde más lo necesita y quizás donde más lo necesitan es en un entorno hospitalario) ( $\mathrm{N}$ : a mí me aporta frescura porque incluso a veces ellos nos 
aportan más nosotros, yo me vengo como más renovada). Más aún, como destaca la maestra, a los docentes les sirve para dar clase de una manera totalmente distinta a como acostumbran en la escuela: interrupciones constantemente de niños que entran o salen para hacerse pruebas médicas, niños encamados o incapacitados de movimiento.

\subsection{La organización y gestión}

En lo que se refiere a la organización del proyecto, al inicio de cada curso escolar se avisa a todos los centros de la comunidad autónoma del plazo de inscripción en el proyecto y los docentes que deseen participar deben rellenar un formulario que ha de firmar el director de su centro y reenviar a la organización. Si bien, se observa un desconocimiento por parte de los docentes de la Región por lo que el flujo de información no está siendo eficaz (N: yo me he encontrado compañeros que dicen que no sabían nada del aula hospitalaria. Entonces los maestros que vamos o que estamos ya varios años yendo, intentamos en la medida de lo posible informar para que cada vez haya más compañeros y se puedan apuntar cada vez más). Una vez establecido el calendario se realiza una reunión con el objetivo de que los maestros que asisten por primera vez estén informados del proceso a seguir, el eje temático a abordar, el tipo de actividades a realizar y duración, la forma en la que se hacen, los permisos y documentos a cumplimentar ( $\mathrm{N}$ : se hace una reunión a principio de curso de todos los maestros que van a participar en las aulas hospitalarias. Normalmente se hace para aquella gente que nunca ha ido al aula hospitalaria pues para informarle, las actividades que se hacen, cómo se hacen, en los tiempos que se hacen y los permisos).

En relación con el eje temático que rige la práctica docente hospitalaria, se selecciona anualmente una temática que resulta interesante para los niños aunque los voluntarios cuentan con cierta libertad (R: cada uno tiene libertad si quiere para seguir el eje temático o para no seguirlo). Asimismo, las distintas festividades que se celebran en la escuela durante el calendario escolar también adquieren protagonismo en las aulas hospitalarias realizando sesiones musicales en torno a Halloween, Navidad o el Día de la Paz. Atendiendo a las memorias del programa es pertinente enumerar los ejes temáticos que se han seguido: el cine, los musicales, los Derechos del niño hospitalizado, hábitos saludables, el medio ambiente, los sueños, el miedo, los viajes, Cartagineses y Romanos, astronomía, el circo, reciclaje, caballeros y castillos, el universo, emociones, la primavera, museos y también, los cuentos.

Esta organización previa anual puede tener una desventaja. La planificación a lo largo de todo un curso escolar conlleva que un docente no pueda acudir a la sesión que tenía prevista porque sus circunstancias han cambiado. En ese caso, no puede cubrirle otro maestro al ser conocedora la Consejería de quién y cuándo va a acudir al aula hospitalaria quedando así descubierta la sesión (R: esto al principio en 2004 no estaba así, pero ahora ya cuando se elabora a principio de curso en septiembre el calendario de a qué músico le toca ir cada viernes, eso llega a la conserjería y tú tienes como un permiso para desplazarte el día que te toca, pero no puedes desplazarte a ese centro el día que no te toca). De ahí, que inscribirse por parejas en las distintas sesiones sea un punto fuerte del proyecto, dado que si se da esta circunstancia de que un voluntario no pueda acudir, siempre quedaría otro maestro para poder llevar a cabo la sesión. De acuerdo a los datos obtenidos del blog, existe una mayor presencia de maestras (57.42\%) que de maestros (42.58\%). Además, hay que mencionar que el $33,82 \%$ de las sesiones la impartió un maestro en solitario mientras que el $65.29 \%$ en parejas y solo el $0.59 \%$ en grupos de tres y el $0.29 \%$ en grupos de cuatro (véase figura 1 ). 


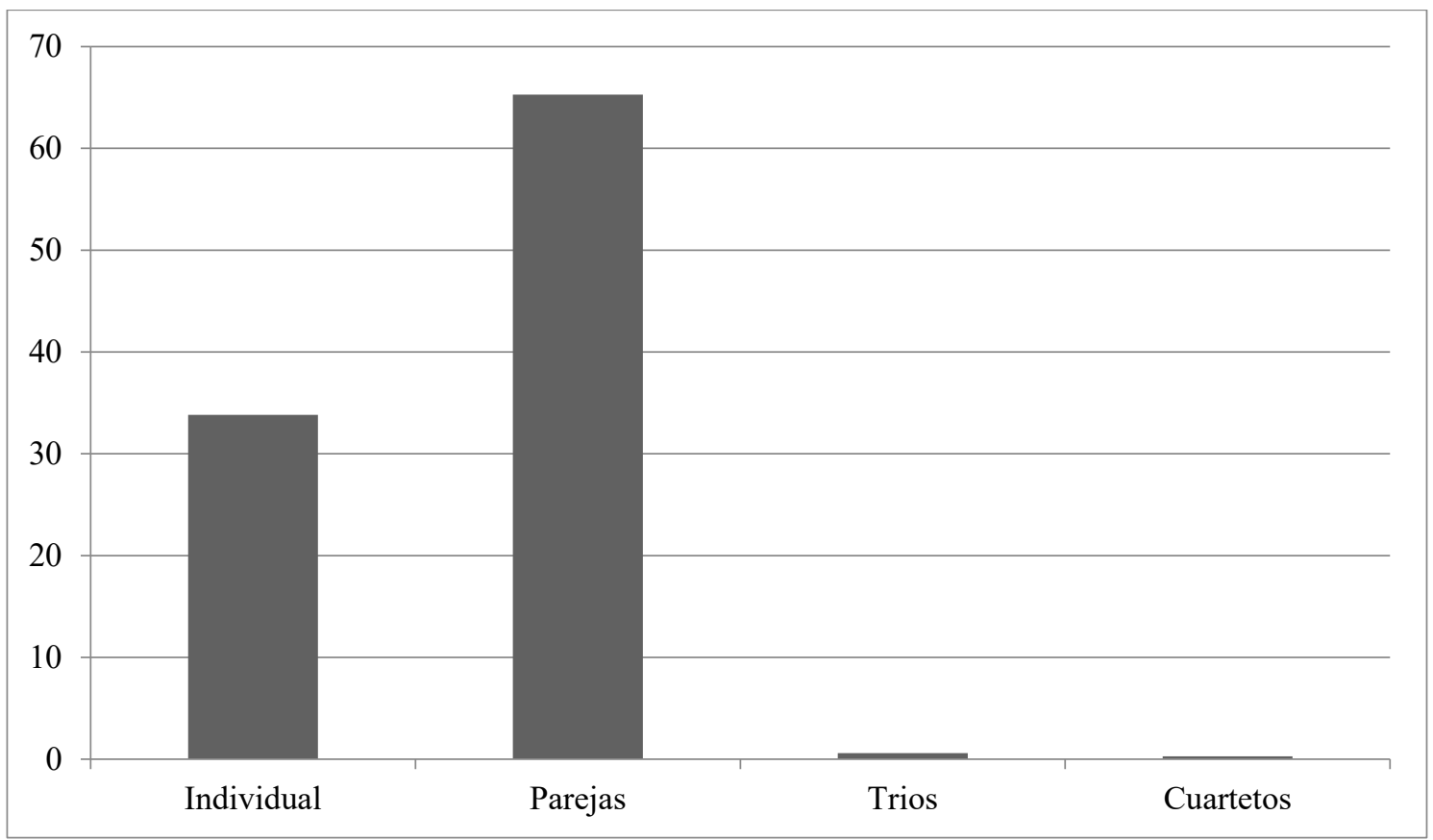

Figura 1. Número de docentes por sesión.

Como añadido, también hay que resaltar que en ocasiones acuden a las sesiones de música colaboradores y profesores del conservatorio aunque estos lo hacen a modo de colaboración y en su tiempo libre. También, se ha de dejar constancia que el alumnado universitario que cursa estudios de Grado en Maestro de Primaria y que está tutorizados durante su periodo de prácticas escolares por un docente que tiene previsto impartir una sesión en el aula hospitalaria debe también desplazarse junto a este.

No obstante, acerca del número de maestros participantes, el primer año que empezó el proyecto, el coordinador, Roberto Pujol, iba solo a las sesiones y no entraba al aula nadie más. Daba él solo las clases, no había más maestros de música que participasen otras semanas, únicamente le ayudaban un par de maestros titulares del aula hospitalaria. Al finalizar el primer año de la puesta en marcha del proyecto era Roberto junto a otros maestros quién acudía a las sesiones, pero siempre estaba presente el coordinador (R: yo estaba con el músico al que le tocara y ahora ya, desde que el proyecto ha ido creciendo, que hay más de ochenta y pico maestros metidos en el asunto no).

Tal y como afirma Roberto, en una sesión es obligatorio que se encuentren presentes el voluntario y los maestros titulares del aula hospitalaria, ( $R$ : si es en pediatría pues las profesoras del aula de pediatría, si es en oncología pues las profesoras del aula de oncología) y personal sanitario como celadores o enfermeros por si se da el caso de que algún niño lo necesita o por si se lo tienen que llevar a hacerle alguna prueba médica esté localizado. Como comentó Natalia, los familiares de los alumnos también participan en las sesiones con sus hijos ( $\mathrm{N}$ : la verdad que la participación de los padres es importantísima para ayudar a los alumnos, para coger los instrumentos, para todo).

\subsection{La práctica musical en las aulas hospitalarias}

Como bien señala Carrasco (2008) en su investigación, la maestra del aula hospitalaria, es decir, no los maestros del proyecto de música, es la encargada de pasar por las habitaciones informando diariamente a los niños que van a hacer cada día. Coincidiendo con las sesiones musicales, la labor de la docente es la de informar y animar así como conocer el estado anímico de cada uno de los niños de cara a que los voluntarios den una mejor respuesta a las necesidades de los mismos. 
La casuística existente en una planta hospitalaria supone que no todas las sesiones se puedan realizar del mismo. Así pues, de los datos obtenidos del análisis documental se concluye que el $69,44 \%$ de las sesiones se han realizado en un aula con varios niños mientras que únicamente un $6,82 \%$ solo se llevaron a cabo en las habitaciones con carácter individualizado. Si bien, el $23,74 \%$ fueron mixtas, es decir, los voluntarios impartieron parte de su jornada con un grupo de alumnos en el aula y también pasaron por las habitaciones realizando pequeñas sesiones individualizadas. Si bien, estos porcentajes varían dependiendo de la planta. En el caso de Oncología las sesiones mixtas están más presentes a consecuencia del estado de los niños donde el docente visita con más frecuencia las habitaciones (Tabla 1).

Tabla 1.

Sesiones realizadas en los distintos espacios

\begin{tabular}{ccccc}
\hline \multirow{2}{*}{ Espacio } & \multicolumn{2}{c}{ Hospital Virgen de la Arrixaca } & Hospital Santa Lucía & Total \\
\cline { 2 - 5 } & Oncología & Pediatría & Pediatría & Total \\
\hline Aula hospital & $42 \%$ & $86,06 \%$ & $77,94 \%$ & $69,44 \%$ \\
\hline Habitaciones & $16 \%$ & $0 \%$ & $0 \%$ & $6,82 \%$ \\
\hline Mixta & $58 \%$ & $13,94 \%$ & $22,94 \%$ & $23,78 \%$ \\
\hline
\end{tabular}

La ubicación para la realización de las sesiones está muy condicionada por dos factores: la movilidad y el estado de ánimo. En relación con el primer factor, los niños que se encuentran encamados o muy débiles para poder desplazarse no acuden al aula recibiendo la sesión dentro de la propia habitación sin un tiempo establecido, es decir, la duración de la misma depende de la respuesta del niño ( $\mathrm{N}$ : te reciben con una sonrisa pero a lo mejor hay críos que están más "malicos" y tienes que esforzarte un poquito más para sacarle algo porque están anímicamente muy mal). Esta situación es más frecuente en la planta de Oncología donde las sesiones mixtas ascienden a un total del $42 \%$ y las realizadas solo en habitaciones individuales a un $16 \%$.

Si bien, siempre que se puedan desplazar al aula aunque sea en silla de ruedas es preferible con el objetivo de favorecer así el proceso de socialización y evitar el aislamiento (Caballero, 2007). En el caso de que la sesión se desarrolle en el aula del hospital, los alumnos acuden dependiendo de sus patologías (aula de oncología, cirugía o pediatría). En cuanto a la distribución de los niños, se sientan todos juntos sin importar sus edades. El alumnado que entra al aula en sillas de ruedas se sienta en la parte de delante, los que llevan un gotero y un pie para su sujeción normalmente se sitúan por los lados de la clase al tener que conectar con frecuencia los monitores a los enchufes. Aun así, no existe una posición establecida al variar continuamente el alumnado, al igual que la entrada y salida al aula durante el tiempo que dure la sesión ( $\mathrm{R}$ : hay interrupciones continuas, de un crio que se lo tienen que llevar porque viene un médico y le tienen que hacer una prueba, o tú estás dando clase y viene alguien porque le tiene que pinchar en la vía una medicación. No es una sesión como en el colegio).

En cuanto a los criterios metodológicos, el proyecto plantea que en las sesiones el rol del niño sea participativo y activo; la realización de actividades que lleven implícito conceptos musicales; combinar actividades musicales diversas (canto, movimiento, lenguaje musical, instrumentación y audición) e introducir el juego.

Tal y como afirma el coordinador del proyecto, los maestros tienen la libertad de planificar las sesiones como quieran, proponer las actividades que estimen convenientes pero siempre se 
recomienda que planifiquen actividades variadas, cortas, adaptables a cualquier edad y con el eje temático preestablecido (R: porque el problema que tiene el hospital es que imagínate que yo hoy me he quedado en el colegio con una actividad a medias, pero yo a estos críos los tengo la semana que viene, pero en el hospital a la semana siguiente no tengo a los mismos críos porque alli van cambiando).

Tal como muestra la figura 1, las sesiones suelen combinar al menos actividades de dos tipos de disciplinas musicales siendo la instrumentación la más presente en las sesiones $(44,20 \%)$, seguida del canto $(20,22 \%)$, la audición $(13,64 \%)$, el lenguaje musical $(11,91 \%)$ y el movimiento $(10,03 \%)$.

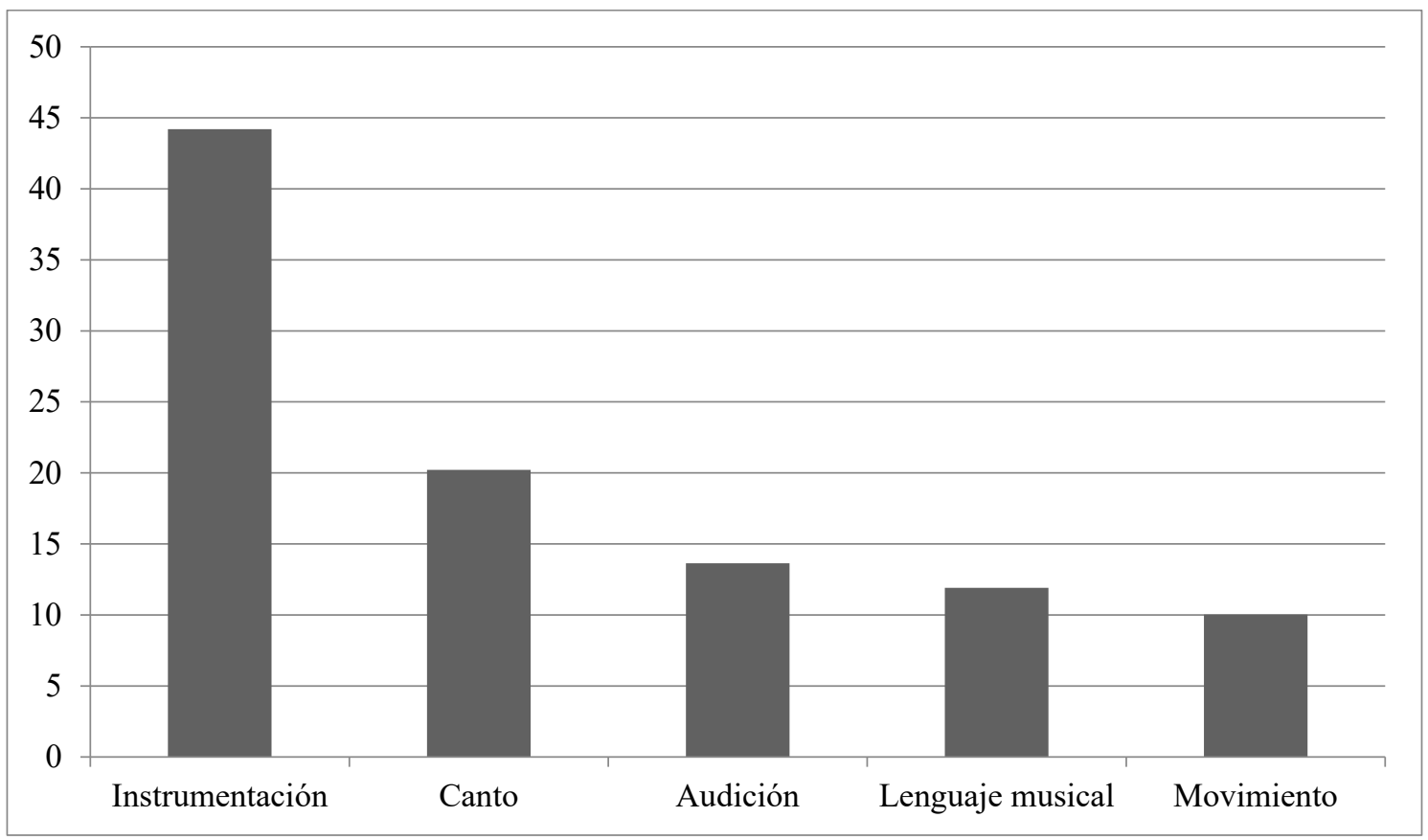

Figura 2. Presencia de las disciplina musicales en el total de sesiones.

Las actividades de instrumentación se orientan a la presentación, reconocimiento y manipulación de instrumentos, efectos sonoros, juegos de discriminación tímbrica, uso y construcción de cotidiafonos u objetos sonoros. Según el coordinador, los instrumentos que más se utilizan son los instrumentos de altura indeterminada (PAI): pandero $(26,18 \%)$, maracas $(21,76 \%)$, triángulo $(16,47 \%)$, pandereta $(14,41 \%)$, caja china $(10,29 \%)$, claves $(9,41 \%)$. También tienen una presencia los boomwhackers $(6,76 \%)$ y las campanas sonoras $(30,59 \%)$ dentro de los de altura determinada (PAD), siendo estas últimas el instrumento más empleado en las sesiones. Se debe a la sencillez y comodidad para tocarlas al solo tener que pulsar un botón y su riqueza sonora. Además, su tamaño pequeño permite un fácil traslado por las habitaciones (R: es una manera muy rápida de hacer música y da muchas posibilidades porque puedes trabajar por colores, por números, por notas. Es una forma muy rápida de hacer una actividad y también que ellos enseguida se animen porque se ven ahí haciendo música en un momento).

Por otra parte, las de canto suponen la realización de pequeñas canciones cortas y exploración de las posibilidades sonoras de la propia voz mientras que las actividades de percepción auditiva se orientan a la escucha de grupos corales o pequeños conciertos. El lenguaje musical estuvo presente en setenta y seis sesiones a través de musicogramas, ejecución de ritmos y percusiones corporales orientadas a trabajar el pulso, acento, ritmo, discriminación de ruido y silencio así como los parámetros del sonido (timbre, intensidad, duración y altura). Por último, las actividades de movimiento y danza fueron las menos numerosas a consecuencia de las 
limitaciones de movimiento que el alumnado hospitalizado suele tener. Aun así en un total de 64 sesiones se llevó a cabo el trabajo con gestos y movimientos para el acompañamiento de canciones, danzas sencillas y dramatización de cuentos.

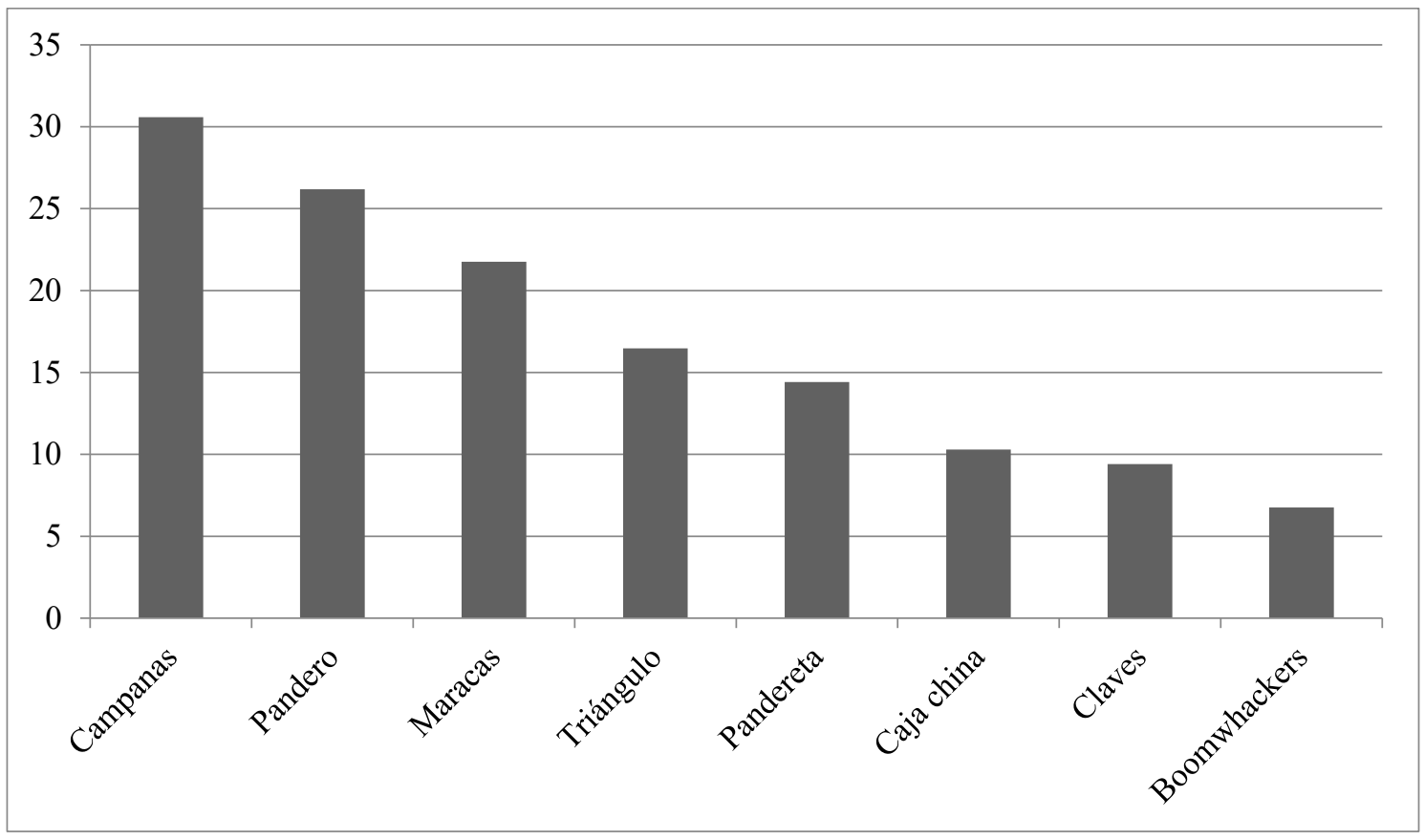

Figura 3. Instrumentos más presentes empleados en las sesiones.

En relación con los recursos materiales se ha de dejar constancia que cuando se inició el proyecto en 2004 el aula hospitalaria no contaba con apenas recursos ( $N$ : había muchos menos recursos. Pero conforme han ido pasando los años, hay muchísimos instrumentos). Así pues, el actual coordinador tenía que llevar y transportar hasta el aula los materiales e instrumentos por su cuenta. Sin embargo, hoy todas las aulas disponen de una pizarra digital, proyector, ordenador, altavoces e instrumentos de música ( $\mathrm{N}$ : ha mejorado muchísimo en la dotación y hay muchísimos recursos que antes no, que había también cosas, pero no la cantidad y la diversidad que hay ahora). Además, todos los años se compran instrumentos nuevos porque algunos se estropean o se amplían los ya existentes ( $\mathrm{N}$ : ellos hacen todo lo posible por comprarlo o porque la próxima sesión lo tengas, en eso no hay problema) ( $R$ : cuando alguien quiere utilizar algo muy específico porque lo necesita, se lo lleva él de su aula o hace una petición).

\subsection{La evaluación: problemas y soluciones}

El proyecto se somete a una doble evaluación. Por un lado, se realiza semanalmente una evaluación de las sesiones puntuales por parte de los docentes que la han impartido ( $R$ : estamos obligados a rellenar una ficha que tú la llevas un poco al principio con lo que pretendes hacer, pero claro, de lo que pretendes hacer a lo que luego haces puede haber un cambio. Entonces luego al final se termina de completar la ficha y hay una evaluación, sobre todo una evaluación a nivel emocional). El factor emocional ocupa un lugar primordial en ella haciendo alusión al objetivo principal del proyecto. Esta evaluación se realiza mediante una ficha establecida que envían a las maestras titulares del aula hospitalaria y en la que se contemplan aspectos diversos junto a una pequeña reflexión personal de la sesión ( $\mathrm{N}$ : los alumnos que hemos tenido, las edades que han comprendido, si han entrado padres, qué alumnos han estado más receptivos, cuáles no, si la sesión ha ido bien, si ha ido regular, si la participación ha sido elevada). 
Al finalizar el curso académico se procede a la evaluación del proyecto y a la elaboración de una memoria donde se concluye si se han o no alcanzado los objetivos, aspectos de mejora, problemáticas, futuras líneas, necesidades materiales que se han de suplir para el próximo curso, así como cualquier aspecto considerado esencial de cara a mejorar el proyecto con vistas al próximo curso.

Tras analizar las memorias, el proyecto ha encontrado a lo largo de sus dieciséis años de trayectoria problemáticas de diversa índole. Entre ellas se encontró la imposibilidad de continuar con el proyecto en el Hospital Reina Sofía de Murcia por incompatibilidad horaria entre las terapias de los niños y el horario previsto para las sesiones musicales. También, se ha aludido a que el inicio de las sesiones en ocasiones es difícil al no responder activamente los participantes tal como se podría esperar (R: viene de su habitación, con su problemática, y a lo mejor viene y acaba de vomitar y el niño no tiene ganas de estar allí). Otra problemática es la falta de recursos materiales cuando la sesión se lleva a cabo en una habitación en vez de en el aula a consecuencia de la falta de un proyector o determinados instrumentos más pesados como puede ser el teclado que emplean algunos docentes para acompañar canciones ( $\mathrm{N}$ : $a$ veces llevamos un carro con un portátil y un altavoz, entonces sí que ponemos las canciones o el musicograma en el ordenador, pero claro, evidentemente tampoco podemos estar arrastrando muchísimos instrumentos). Por último, un problema surgido durante los primeros años del proyecto fue consecuencia de la metodología empleada en las sesiones cuando eran realizadas por profesores del conservatorio y no por maestros de música. Se observó una tendencia a que estos docentes que impartían docencia en el conservatorio realizaban "pequeños conciertos" a través de la interpretación de pequeñas piezas con su instrumento en vez de involucrar al alumnado en la práctica musical. Dicho de otro modo, el alumnado hospitalizado funcionaba como agente pasivo y como un mero oyente (R: Alguno confundió esto como que iba al hospital y allí soltaba y empezaba a tocar como si estuviese dando un concierto. Lo que se intenta es que, aunque uno aporte lo que sabe tocar en algún momento de la clase, sean los críos los que participen).

\section{Conclusiones}

Para concluir, es necesario hacer una mención expresa a la importancia de este estudio que pretende, más allá de conocer el proyecto de Música los Viernes y su funcionamiento, darlo a conocer a la comunidad educativa como una buena práctica que debe ser tenida en cuenta en otros contextos geográficos. Los objetivos que se plantea, ya sean emocionales o musicales, alcanzados curso tras curso académico avalan el éxito de esta propuesta que desde 2004 se ha llevado a cabo de manera ininterrumpida y prosigue aún hoy. Una acción puntual e individual que pasó en un año a institucionalizarse gracias al apoyo y su vinculación con la Conserjería de Educación de la Región de Murcia. A pesar de todo ello, este proyecto a pesar de su impacto en la comunidad infantil es a la fecha un desconocido dentro de la propia comunidad autónoma en la que se está desarrollando.

Analizados los resultados se constata como el proyecto objeto de estudio contribuye a mitigar el estrés de los alumnos hospitalizados independientemente de la duración de su hospitalización (Ariza, 2014). Además, de esta manera los alumnos son capaces de olvidar los problemas e inquietudes que conlleva el ingreso en el hospital pudiendo disfrutar tanto de las sesiones musicales, como de sus compañeros y maestros (Mendoza, 2019).

Así mismo, como ya se ha dicho, entre los 5 tipos de actividades utilizadas en las sesiones, las más utilizadas fueron las de instrumentación apareciendo en un total de $44,20 \%$ de las sesiones debido a que se apuesta porque el alumno tenga un papel activo y participe en estas, no siendo el profesor el que tenga el protagonismo en las mismas. No obstante, coincidiendo 
con la afirmación de Caballero (2007), siempre se tienen que llevar planteadas varios tipos de actividades para que las puedan realizar cualquier alumno.

En relación con los objetivos que persigue el programa de música, de los diez establecidos solo uno de ellos está relacionado con solventar los problemas emocionales que les surgen a los niños durante el periodo de hospitalización. En cambio, los nueve restantes, se orientan a la enseñanza y práctica de la educación musical. Además, sabiendo los beneficios que aporta a los alumnos hospitalizados la música, se ha destacado que era más prioritario la mejora emocional que los avances académicos coincidiendo con distintos autores (Mendoza, 2019; Carrasco, 2012; Todres, 2006; Barrera, Rykov y Doyle, 2002).

Este trabajo debe ser el germen para proseguir con futuras líneas de investigación vinculadas a la presencia de la música en las aulas hospitalarias. Concretamente, sería pertinente, dado que este estudio da la visión del funcionamiento del proyecto desde una perspectiva más burocrática así como desde la percepción de la coordinación y los voluntarios participantes, conocer también la visión de quienes se benefician del mismo (niños y familias). Igualmente, aunque en España no se desarrolla ninguna experiencia similar, puede ser de interés conocer sí existen otras experiencias similares fuera de las fronteras y compararlos entre sí con el objetivo de introducir mejoras a través de otras buenas prácticas similares.

\section{Referencias bibliográficas}

Arias, F.G. (2019). Citación de fuentes documentales y escogencia de informantes: un estudio cualitativo de las razones expuestas por investigadores Venezolanos. e-Ciencias de la Información, 9(1), 1-24.

Ariza, L. (2014). El aula hospitalaria: una pedagogía en positivo. Revista Educación y Futuro Digital, (9), 54-59.

Barrera, M., Rykov, M. y Doyle, S. (2002). The effects of interactive music therapy on hospitalized childrenwith cancer: a pilot study. Psyco-oncology, 11(5), 379-388.

Barros, J., Praxedes, J., Pereira, S., Gabrieli, A., Terezinha, L. Z., Netto, C., Luize, E., y Demeneck, R. (2019). Música no hospital: Promoção da saúde na oncologia. Revista Brasileira Em Promoção Da Saúde, 32, 1-8.

Caballero, S. (2007). El aula hospitalaria un camino a la educación inclusiva. Investigación Educativa, 11(19), 153-161.

Cárdenas, R. y López, F. (2005). Hacia la construcción de un modelo social de la Pedagogía Hospitalaria. Pedagogía social: revista interuniversitaria, (12-13), 59-70.

Carrasco, P. (2008). Estudio del valor terapéutico de la literatura infantil en niños hospitalizados. (Tesis Doctoral). Universidad de Murcia, España.

Carrasco, P. (2012). La actuación Educativa en las Aulas Hospitalarias.

Clauso, A. (1993). Análisis documental: el análisis formal. Revista General de Información y Documentación, 3(1), 11-19.

Denzin, N. K. y Lincoln, Y. S. 1994. Introduction: entering the field of qualitative research, en N. K. Denzin e Y. S. Lincoln (eds.), Handbook of Qualitative Research. Thousand Oaks, California: Sage.

Drake, L., Ben-Yelun, F. y Del Olmo, M. (2017). Musicoterapia en una UCl pediátrica: El uso de la música como emergente emocional en el proceso de intervención con niños en situación de riesgo. Revista de Investigación en Musicoterapia, 1, 14-31. 
Fernández, M. (2000). La pedagogía hospitalaria y el pedagogo hospitalario. Tabanque: revista pedagógica, (15), 139-150.

García, A. (2012). Escuelas hospitalarias en España, Suecia y Argentina: evolución y situación contemporánea de las instituciones comprometidas con la educación del niño y adolescente en situación de enfermedad (Tesis Doctoral). Universidad Autónoma de Madrid, España.

Jacobsen, J. (1997). Pet Therapy: A study of the use of companion animals in selected therapies. Pikes Peak. Colorado Spring, Colorado.

Ley 13/1982, de 7 de abril, de integración social de los minusválidos, núm.103, 11106-11112.

Mendoza, M. (2019). Relevancia del juego y actividades en las aulas hospitalarias. Educación: Revista de la Facultad de Ciencias de la Educación, 25(1), 49-54.

Peña, D. (2015). Validación por expertos del programa trufas de Educación Asistida con Perros para la prevención del acoso escolar (Trabajo Fin de Máster). Universidad Complutense, Madrid.

Real Decreto $1 / 2013$, de 29 de noviembre, por el que se aprueba el Texto Refundido de la Ley General de derechos de las personas con discapacidad y de su inclusión social, núm. 289, 956335-96673.

Real Decreto 334/1985, de 6 de marzo, de ordenación de la Educación Especial, núm 65, 1-12.

Real Decreto 696/1995, de 28 de abril, de ordenación de la educación de los alumnos con necesidades educativas especiales, núm 131, 1-13.

Riquelme, F., Cerdán, L. y Sánchez, J. (2012). Proyecto "Martes con arte". La actuación Educativa en las Aulas Hospitalarias.

Rodríguez, G., Gil, J. y García, E. (1999). Metodología de la investigación cualitativa. Málaga, España: Aljibe.

Serradas, M. (2010). Colaboración interdisciplinaria en la atención del niño con cáncer y su familia en ambientes hospitalarios. Revista Venezolana de Oncología, 22(3), 174-186.

Serrano, J. y Prendes, M.P. (2015). Integración de TIC en aulas hospitalarias como recursos para la mejora de los procesos educativos. Estudios Sobre Educación, 28, 187-210.

Todres, D. (2006). Music is medicine for the heart. Jornal de Pediatria, 82(3), 166-168.

Vila, J. y Barrena, J. (2012). Atención a la diversidad y compensación educativa en el aula hospitalaria. TRANCES: Revista de Transmisión del Conocimiento Educativo y de la Salud, 4(6), 461-478.

Webmaster Plataforma (2011). Blog del Equipo de Atención Educativa Hospitalaria y Domiciliaria Región de Murcia. E.A.E.H.D. Región de Murcia. [Blog]. Recuperado de https://www.murciaeduca.es/eaehd/bitacora/ 